\title{
LETTERS
}

\section{Mechanisms of dyspnoea relief following radiation treatment in a patient with severe COPD}

\section{To the Editors:}

Chronic obstructive pulmonary disease (COPD) and lung cancer coexist in many smokers. In those with more advanced COPD in whom surgical resection is contraindicated, palliative or curative radiotherapy may be considered. The effects of radiation treatment on respiratory physiology and functional status in patients with background COPD are highly variable and difficult to predict [1]. Radiation-associated inflammatory injury to airways, lung parenchyma and the pulmonary vasculature can lead to further respiratory impairment, including disruption of pulmonary gas exchange with consequent clinical deterioration. The decision to proceed with radiotherapy must be carefully weighed and based on individualised risk-benefit analysis, especially in those patients with severe COPD. Contrary to expectation, there are anecdotal reports of patients with COPD who experience symptomatic improvement following radiation treatment, but the mechanisms remain unknown [2].

Our patient was a 70 yr-old female with a 50-pack-yr smoking history who had documented progressive respiratory deterioration despite maximal medical therapy and pulmonary rehabilitation. Pulmonary function tests (PFTs) prior to treatment indicated very severe airway obstruction (forced expiratory volume in $1 \mathrm{~s}$ (FEV1) $34 \%$ predicted) and lung hyperinflation (total lung capacity (TLC) 155\% pred), and diffuse centrilobular emphysema was evident on computed tomography (CT) scan, although diffusing capacity of the lung for carbon monoxide $(D \mathrm{~L}, \mathrm{CO})$ was relatively preserved (13.6 $\mathrm{mL} \cdot \mathrm{min}^{-1} \cdot \mathrm{mmHg}^{-1}, 73 \%$ pred). CT scanning fortuitously identified a suspicious spiculated nodule $(10.5 \times 11 \mathrm{~mm})$ in the patient's right middle lobe, which increased in size by $2 \mathrm{~mm}$ over 11 months with no evidence of airway obstruction or atelectasis. Subsequent positron emission tomography demonstrated that she probably had primary lung cancer in stage Ia (T1aN0M0). Tissue diagnosis was not attempted given the location of the nodule, her fragile respiratory status and the high risk of pneumothorax. Our patient had severe dyspnoea and disability (Medical Research Council (MRC) rating IV).

Surgery was precluded due to the patient's poor lung function but given the small size of the lesion and the possibility of cure, radical radiation treatment was recommended with the aim to minimise the total radiation dose. The possibility of amplifying her already severe dyspnoea and of her requiring lifelong oxygen therapy was presented, and she consented to treatment. Radiation was administered at a dose of $60 \mathrm{~Gy}$ in 15 fractions, 5 days $\cdot$ week $^{-1}$ for 3 weeks using a linear accelerator. Three-dimensional conformal radiation treatment planning was used to target the nodule in the right middle lobe. Over the 2 months following her radiation treatment, the patient reported improvement in dyspnoea, which was first noticeable after the second radiation session: 8 months after treatment, her MRC rating was II and she claimed that this treatment had given her "a new life". Her requirement for rescue bronchodilator diminished in tandem with the improvement in dyspnoea. She did not report any side-effects from the radiation, and incidentally noted abatement of her habitual cough and sputum production. Repeat CT scan 6 months after radiotherapy demonstrated that the nodule remained stable and that there was a decrease in right lung volume by $227 \mathrm{~mL}$, as determined using a GE Advantage Workstation (version 4.3; GE Healthcare, Little Chalfont, UK). There was no evidence of atelectasis, radiation pneumonitis or fibrotic scarring, and no change in emphysema distribution following radiation.

Compared with the baseline PFTs 8 months earlier, PFTs performed after radiotherapy showed a reduction in static lung hyperinflation: TLC, functional residual capacity (FRC) and residual volume (RV) decreased by 14, 24 and 40\%, respectively, and inspiratory capacity (IC) and forced vital capacity (FVC) increased by 49 and 63\%, respectively. FEV1 increased by $380 \mathrm{~mL}(58 \%)$ but the FEV1/FVC ratio remained unchanged at 0.32 . DL,CO decreased by $27 \%$.

We used the results of the patient's previous cardiopulmonary exercise test conducted $\sim 2.5$ yrs prior to radiation therapy as a baseline reference. These test results probably underestimated her poor functional status immediately prior to radiotherapy but nevertheless served as a useful comparator for examining mechanisms of benefit. An identical incremental cycle exercise protocol was used for both tests in the same testing facility; maximal motivational effort was expended on both occasions and the patient remained on her usual long-acting bronchodilators prior to each exercise test. Compared with the reference test: 1) dyspnoea intensity ratings were uniformly lower and the primary locus of sensory limitation changed from breathing difficulty (described as a feeling of suffocation and inspiratory difficulty) to leg discomfort; 2) peak work rate and peak oxygen uptake increased by $20 \mathrm{~W}(67 \%)$ and $4.1 \mathrm{~mL} \cdot \mathrm{kg}^{-1} \cdot \mathrm{min}^{-1}(37 \%)$, respectively; 3) the ventilatory equivalents for oxygen and carbon dioxide decreased throughout exercise, indicating improved efficacy of ventilation; and 4) breathing pattern became deeper and slower in conjunction with increased IC during rest and exercise. Arterial oxygen saturation and cardiovascular responses (data not shown) were similar at peak exercise. Sensory, ventilatory and breathing pattern responses can be seen in figure 1.

The role of radical radiation therapy in those patients with COPD who are unsuitable for surgery is still uncertain. Several 

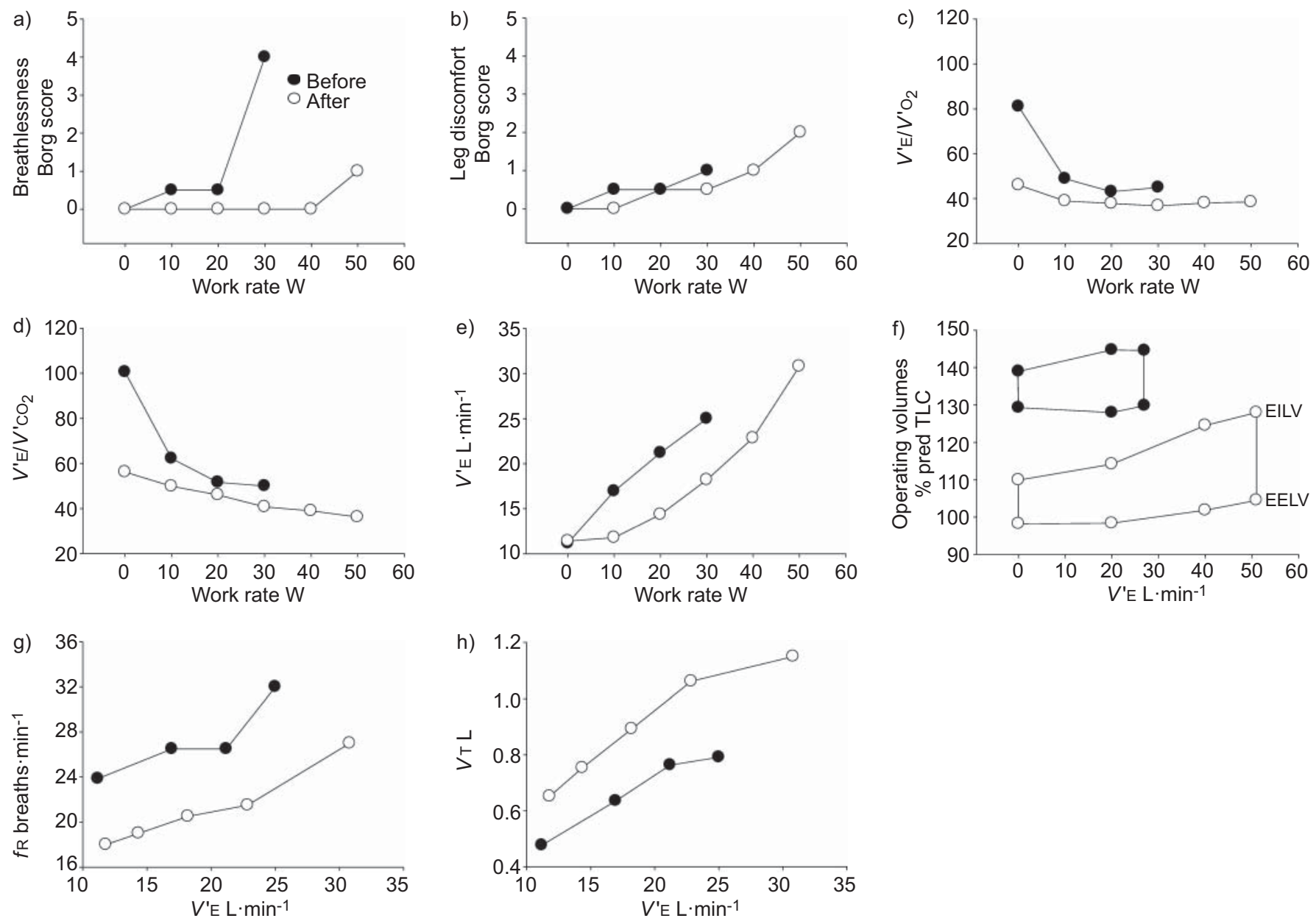

FIGURE 1. Borg scale a) breathlessness and b) leg discomfort, and ventilatory equivalents for c) oxygen (oxygen uptake; $V^{\prime} \mathrm{O}_{2}$ ) and d) carbon dioxide (carbon dioxide production; $\left.V^{\prime} \mathrm{CO}_{2}\right)$, e) minute ventilation $\left(V^{\prime} \mathrm{E}\right)$, f) operating volumes, g) respiratory frequency (fR), and h) tidal volume ( $(\mathrm{V})$ in response to exercise before and after radiation treatment. \% pred: \% predicted; TLC: total lung capacity; EILV: end-inspiratory lung volume; EELV: end-expiratory lung volume.

studies examined the effect of radiation on pulmonary function, with mixed results. The one consistent finding has been a decrease in $D \mathrm{~L}, \mathrm{CO}$, which is thought to be due to loss of alveolar surface area and thickening of the intra-alveolar septa, and which has been similar to the decrease in diffusing capacity expected after surgical resection [3]. Smaller but consistent decreases in TLC and FVC have also been observed. The change in FEV1 has been variable, ranging from a small improvement to a mild decrease, with several studies showing no change $[1,3,4]$. Interestingly, a small study by BousHY et al. [5] demonstrated an increase in FEV1 of $160 \mathrm{~mL}$ only in those patients who had severe baseline COPD (FEV1 $\leqslant 50 \%$ pred).

The effect of radiotherapy on dyspnoea intensity in COPD is unknown and is probably highly variable. However, clinical experience tells us that some individuals derive symptomatic benefit. In fact, AXFORD et al. [2] proposed low-dose radiation as a dyspnoea-relieving intervention for patients with severe COPD (FEV $1<30 \%$ pred) with refractory dyspnoea. In that uncontrolled study, 10 patients received an average of 1,600 rads (16 Gy) to one or two areas of lung with high ventilation-perfusion ratios (measured using radioactive iodine). Dramatic subjective improvement in dyspnoea and walking tolerance occurred in the majority of patients over an average of 13 weeks post-radiation. The authors speculated that reduction in dynamic airway compression was a possible contributing factor.

The putative explanation for improved dyspnoea following radiotherapy in our patient is improved respiratory mechanics and reduced ventilatory requirements. The reduced TLC, FRC and RV (by 14, 24 and 40\%, respectively), in the absence of any change in maintenance bronchodilator therapy, must ultimately reflect changes in elastic properties of the lung (increased elastic lung recoil pressures). The other known determinants of these static lung volumes (chest wall compliance and inspiratory muscle strength) were unlikely to be affected by radiotherapy. Reduced lung hyperinflation of the magnitude seen here, which is comparable to that previously reported following pharmacological or surgical lung volume reduction, is associated with improvement in the operating characteristics and contractile strength of the respiratory muscles [6, 7]. In neurophysiological terms, lung volume reduction (and reduced ventilatory demand) leads to a 
diminished central motor output required to drive the respiratory muscles. Thus, the associated perceived effort required to generate a given ventilation during exercise is similarly reduced. The deeper, slower breathing pattern after radiation therapy and delay in the onset of tachypnoea reflect the recruitment of IC both at rest and during exercise as a result of lung volume deflation. Thus, the patient could increase tidal volume and ventilation during exercise to a greater extent than previously, before reaching critical mechanical constraints. For this reason, the onset of intolerable dyspnoea was delayed and the limits of tolerance extended.

Ventilatory requirements also decreased after radiation, presumably reflecting a net improvement in the ventilationperfusion relationship. Thus, measurements of ventilatory inefficiency improved, reflecting more effective carbon dioxide elimination and reduced wasted ventilation. Arterial oxygen saturation was unaffected throughout exercise. The $27 \%$ reduction in $D \mathrm{~L}, \mathrm{CO}$ is a well-documented effect of radiation injury to the pulmonary vasculature and indicates a reduction in the surface area for pulmonary gas exchange.

The clinical decision to offer radical radiotherapy to individuals with severe COPD with early-stage lung carcinoma is often difficult given the known negative consequences of further erosion of an already limited ventilatory reserve. Given the vast pathophysiological heterogeneity of COPD, the impact of targeted radiotherapy is likely to be highly variable. It is conceivable, as our case illustrates, that in some patients with extensive lung hyperinflation and emphysema, radiationinduced alterations in the elastic properties of the lung and in ventilation-perfusion mismatching may actually have favourable effects on dyspnoea and exercise tolerance. Prospective studies to comprehensively characterise COPD phenotypes and measure the effects of radiation on respiratory physiology and patient-centred outcomes are required to better refine selection criteria for radiotherapy in this population.

P. O'Meara*, J.A. Guenette*, N. Raghavan*, N. Amornputtisathaporn*, C.E. deMetz", R.L. Nolan" and D.E. O'Donnell*

*Respiratory Investigation Unit, Dept of Medicine, Queen's University and Kingston General Hospital, "Cancer Centre of
South Eastern Ontario, Kingston General Hospital, and "Dept of Diagnostic Radiology, Kingston General Hospital, Kingston, ON, Canada.

Correspondence: D.E. O'Donnell, Division of Respiratory and Critical Care Medicine, Dept of Medicine, Queen's University, 102 Stuart Street, Kingston, Ontario, K7L-2V6, Canada. E-mail: odonnell@queensu.ca

Support Statement: J.A. Guenette was supported by the John Alexander Stewart Fellowship (Dept of Medicine, Queen's University and Kingston General Hospital, Kingston, ON, Canada) and the Natural Sciences and Engineering Research Council of Canada Post Doctoral Fellowship. N. Raghavan was supported by the John Alexander Stewart Fellowship.

Statement of Interest: A statement of interest for D.E. O'Donnell can be found at www.erj.ersjournals.com/site/ misc/statements.xhtml

\section{REFERENCES}

1 Ghafoori P, Marks LB, Vujaskovic Z, et al. Radiation-induced lung injury. Assessment, management, and prevention. Oncology (Williston Park) 2008; 22: 37-47.

2 Axford AT, Cotes JE, Deeley TJ, et al. Clinical improvement of patients with emphysema after radiotherapy. Thorax 1977; 32: 35-39.

3 Abratt RP, Morgan GW. Lung toxicity following chest irradiation in patients with lung cancer. Lung Cancer 2002; 35: 103-109.

4 Hoffbrand BI, Gillam PM, Heaf PJ. Effect of chronic bronchitis on changes in pulmonary function caused by irradiation of the lungs. Thorax 1965; 20: 303-308.

5 Boushy SF, Helgason AH, North LB. The effect of radiation on the lung and bronchial tree. Am J Roentgenol Radium Ther Nucl Med 1970; 108: 284-292.

6 Laghi F, Jubran A, Topeli A, Fahey PJ, et al. Effect of lung volume reduction surgery on neuromechanical coupling of the diaphragm. Am J Respir Crit Care Med 1998; 157: 475-483.

7 O'Donnell DE, Hamilton AL, Webb KA. Sensory-mechanical relationships during high-intensity, constant-work-rate exercise in COPD. J Appl Physiol 2006; 101: 1025-1035.

DOI: $10.1183 / 09031936.00029411$

\section{Linezolid safety, tolerability and efficacy to treat multidrug- and extensively drug-resistant tuberculosis}

\section{To the Editors:}

Treatment of multidrug-resistant (MDR) tuberculosis (TB) (defined as in vitro resistance to at least isoniazid and rifampicin, the two most potent first-line drugs for TB treatment) and extensively drug-resistant (XDR)-TB (defined as in vitro resistance to isoniazid and rifampicin plus any fluoroquinolone and at least one of the injectable drugs: amikacin, capreomycin or kanamycin) is still a major problem from both a clinical and a public health perspective [1-5].

Treatment outcomes for complicated MDR-TB cases (those with additional resistance beyond isoniazid and rifampicin) and XDR-TB cases being still poor, the need for information on the safety, tolerability and efficacy of other antibiotics that are potentially useful in their treatment is urgent [6-9]. 